\title{
Editorial
}

\section{A Perspective on Research, Education, Practice and Policy in the COVID-19 Era}

Julie A. Jacko, $\mathrm{PhD}^{1}$

\section{Abstract}

\section{Description}

The coronavirus disease 2019 (COVID-19) pandemic has unleashed a global health crisis, precipitating massive efforts aimed at understanding, preventing, preparing for, treating and responding to the novel coronavirus domestically and internationally. Given the profound and ongoing impact of COVID-19, the HCA Healthcare Journal of Medicine organized this special issue of the journal as a mechanism for capturing groundbreaking studies, advances and perspectives that have the potential to shape health and health care for decades to come.

\section{Keywords}

SARS-CoV-2; COVID-19; pandemics; coronavirus infections; disasters

Author affiliations are listed at the end of this article.

Correspondence to: Julie A. Jacko, PhD Professor \& Founding Chair Department of Population Health Science

Dr. Kiran C. Patel College of Allopathic Medicine Nova Southeastern University 3200 South University Drive Fort Lauderdale, FL 33328

(ijacko@nova.edu)
The coronavirus disease 2019 (COVID-19) pandemic has unleashed a global health crisis, precipitating massive efforts aimed at understanding, preventing, preparing for, treating and responding to the novel coronavirus domestically and internationally. As of the time of this writing, 55.6 million people worldwide have been infected with COVID-19, 35.8 million people have recovered and 1.34 million people have died from the virus.'

In addition to its toll on human health and life, there are also incalculable secondary effects of COVID-19, including economic contraction, unemployment, failed business, poverty, hunger, violence, suicide and opioid/substance abuse, all of which have been exacerbated by the pandemic. The pandemic has also upended U.S. health care. Initially, most health systems rapidly activated emergency response plans and cancelled all non-urgent procedures and clinic visits, thus experiencing major negative financial impacts from the pandemic. The nonclinical workforce was often moved to work-from-home arrangements, while virtual communication with patients through the use of telehealth exploded. ${ }^{2}$
As Slotkin et al. marveled early in the pandemic, "the profound disruption in just 12 weeks of an industry, a century in the making, is astounding."2 Health systems around the country have struggled to deal with critical aspects of care delivery, management, decision-making, workforce deployment, communications and operations in response to the COVID-19 pandemic.

Academic medicine has also been strongly impacted. From virtual admission interviews to rapidly converting clinical curricula to virtual delivery overnight, to ensuring that learning objectives are being met across new delivery modalities over time, medical education has had to shift dramatically. These challenges have all given rise to the formulation of novel approaches to the design and delivery of medical education across the country.

Given the profound and ongoing impact of COVID-19, the HCA Healthcare Journal of Medicine organized this special issue of the journal as a mechanism for capturing groundbreaking studies, advances and perspectives that have the potential to shape health and health care for decades to come.

\section{HCA is Healthcare}

\section{www.hcahealthcarejournal.com}

(C) 2020 HCA Physician Services, Inc. d/b/a Emerald Medical Education
HCA Healthcare Journal of Medicine 
Our vision for the special issue was to cast a wide net; not only seeking cases that reported the biological effects of COVID-19 and its causative agents, but also coalescing manuscripts covering a variety of related themes of interest, reflecting the multidimensionality of the impact of the pandemic. In this special issue you will find clinical research, population health research, clinical case reports, medical education advances, quality improvement studies, and humanities pieces, all related to the COVID-19 pandemic. Our commitment to rapid dissemination of these advances through this special issue may pave a way forward to even better adjustment to the post-COVID-19 world, bringing about major changes in the U.S. healthcare system.

The special issue contains a multitude of research articles and case reports; several of which focus on positivity and prevalence, admission rates for those with substance use disorders, remdesivir-warfarin drug interaction, respiratory distress, latent pulmonary embolism and giant cavitary lesion development, and the neuropsychiatric presentation of COVID-19. Advances in medical education also feature prominently. In particular, an obstetrics and gynecology residency program's approach to the pandemic, the use of virtual interviews for residency programs and the urgent integration of virtual reality simulation in medical education are included.

There is also focus on practice management, including articles on conservation of clinical devices, pharmacy services innovations and support for primary care practices. Several esteemed colleagues provided editorials for the issue, including managing fear-based barriers to care and an editorial concerning the conduct of research amidst the pandemic. All of these articles, and more, are provided with the intent to rapidly disseminate the latest scientific advances in clinical care, medical education, practice management and more.

The special issue has been an impressive community effort, borne out of the vision of our Editor-in-Chief, Dr. Bruce Deighton, along with the members of the Editorial Board of the journal, who were active participants in the effort. The issue was also made possible by the many authors and reviewers who de- voted countless hours to ensuring that every contribution included in the issue meets or exceeds the quality standards established for this journal, while being highly relevant to the unique challenges presented by the pandemic. I also wish to acknowledge and thank our special issue guest reviewers, faculty of Nova Southeastern University's Dr. Kiran C. Patel College of Allopathic Medicine, who contributed their time and expertise over several months, enhancing our capacity to process manuscripts through our rigorous peer review process while managing an abundance of submissions to the special issue:

Pran Andhee, M.D.

Rolando DeLeon, M.D., F.A.C.O.G.

Lauren Fine, M.D., FAAAAI

Arkene Levy, Ph.D.

Michael Parker, Ph.D.

Vijay Rajput, M.D., FACP, SFHM

François Sainfort, Ph.D.

Michelle Zhao, Ph.D.

Bringing this issue to fruition has been an incredibly rewarding experience that would not have been possible without the expert knowledge of Graig Donini, our Managing Editor. I offer my deepest gratitude to everyone involved.

Julie A. Jacko, PhD

Special Issue Editor

November 24, 2020

\section{Conflicts of Interest}

The author declares he has no conflicts of interest.

\section{Author Affiliation}

1. Dr. Kiran C. Patel College of Allopathic Medicine, Nova Southeastern University, Fort Lauderdale, FL

\section{References}

1. COVID-19 Dashboard. Johns Hopkins Center for Systems Science and Engineering. Published January 22, 2020. Accessed November 20, 2020. https://coronavirus.jhu.edu/us-map

2. Slotkin JR, Murphy K, Ryu J. How One Health System is Transforming in Response to Covid-19. Harvard Business Review. Published June 11, 2020. https://hbr.org/2020/06/how-one-healthsystem-is-transforming-in-response-to-covid-19 